\title{
Transverse Excitation of Plasmonic Slot Nano-Resonators Embedded in Gold-Coated Microfiber Tips
}

\author{
Ming Ding*, Michalis N. Zervas, Gilberto Brambilla \\ Optoelectronics Research Centre, University of Southampton, Southampton, SO17 1BJ, United Kingdom
}

\begin{abstract}
Nanoscale aperture in thin noble-metal films, with dimensions comparable to the light wavelength, can form plasmonic nano-resonators (PNRs) and show astonishing optical properties leading to enhanced and selective light transmission and confinement. In contrast to conventional optical systems using lenses and mirrors, PNRs can provide hot spots in the near field with dimensions determined mainly by the structure size rather than by the diffraction limit. A number of different nano-structures have been considered and studied in detail for their ability to concentrate light [1]. In this paper, for the first time we study both theoretically and experimentally the transverse excitation of a strongly-coupled 3D plasmonic slot nano-resonator (PSNR) by embedding a rectangular slot nano-cavity in a plasmonic structure formed by a thin-metal-film coated microfiber tip: a schematic of the structure is shown in the inset of Fig. 1(a). Light is launched from the untapered side of the fiber and coupled to the nano-cavity. The various resonances can be identified simply monitoring the reflected light. In the experiment, a PSNR with $400 \mathrm{~nm}$ length, $200 \mathrm{~nm}$ width and $30 \mathrm{~nm}$ depth was inscribed on a $30 \mathrm{~nm}$ goldcoated microfiber tip by focused ion beam (FIB). Light with the polarization state which is perpendicular to the PSNR was launched into the tip and reflection spectra were recorded.

Theoretical analysis was carried out using COMSOL 4.1 Multiphysics to numerically solve Maxwell equations in the frequency domain for the proposed designs. A rectangular PSNR with width $D=400 \mathrm{~nm}$ and length $L=200 \mathrm{~nm}$ was embedded in a conical silica waveguide with $0.9667 \mu \mathrm{m}$ minimum diameter, $1.47 \mu \mathrm{m}$ maximum diameter, $5.33^{\circ}$ taper angle and $30 \mathrm{~nm}$ gold coating layer. Fig. 1(a) shows the transmissivity and the reflectivity of the composite structure with the rectangular PSNR when the input light is $x$ polarized and the inset shows the simulation structure of PSNR. The transmission and reflection spectra show several related dips, which correspond to different type of resonances of the embedded PSNR. The resonance wavelength $1450 \mathrm{~nm}$ with $x$ polarized light gave the biggest enhancement factor $7.24 \times 10^{3}$, which is few times larger than that at the other resonance wavelengths.
\end{abstract}
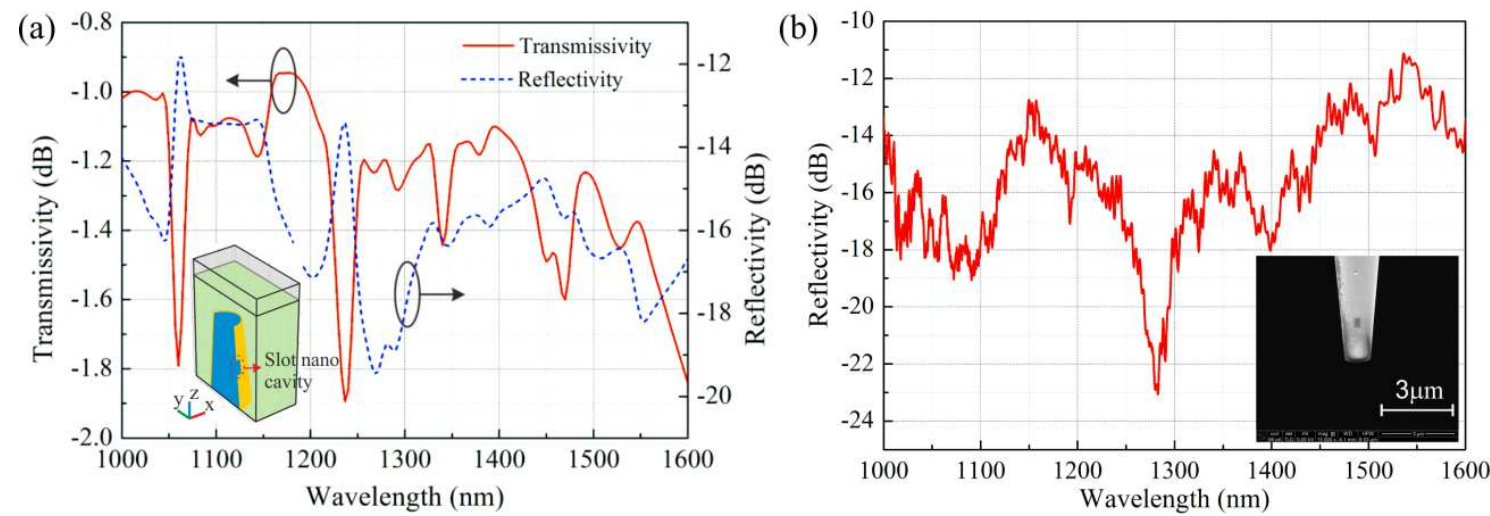

Fig. 1 (a) Transmissivity (red solid) and reflectivity (blue dash) of the rectangular PSNR embedded in the plasmonics fiber tip for $x$ polarized light. Inset: schematic of the PSNR embedded in the plasmonic microfiber tip. (b) Reflectivity spectrum of the sample in the experiment. Inset: SEM image of the plasmonic microfiber tip with a rectangular PSNR.

In order to verify the numerical analysis, a PSNR was inscribed on the surface of a gold-coated microfiber tip using the same geometry of the simulations. The fabrication process involved three main steps: manufacture of optical microfiber tip, deposition of a thin gold layer and FIB nanopatterning. The SEM image of the rectangular PSNR on a plasmonic microfiber tip is shown in the inset of Fig. 1(b). Many resonance features were observed in the reflectivity of the samples recorded at the polarization perpendicular to the PSNR (Fig. 1(b)). The mismatch between experiements and simulations might be related to imperfect taper and gold layer surface and to particles attached to the gold layer surface. Particles induce plasmonic effects even when their size is small. Moreover, part of the gold layer was removed due to the re-deposition of material in the FIB processing and formed additional extremely small nano-cavities. This not only causes plasmonic cavities but also changes the interference condition in the plasmonic microfiber tip. This device can find a wide range of applications such as SERS, optical filtering, spectroscopy and bio-sensing.

\section{References}

[1] B. Lee, I. M. Lee, S. Kim, D. H. Oh, and L. Hesselink, "Review on subwavelength confinement of light with plasmonics," J Mod Optic 57, 1479-1497(2010) 